\title{
The Local Group Census: Planetary nebulae in IC 10, Leo A and Sextans $A^{\star}$
}

\author{
L. Magrini ${ }^{1}$, R. L. M. Corradi ${ }^{2}$, R. Greimel ${ }^{2}$, P. Leisy ${ }^{2,3}$, D. J. Lennon ${ }^{2}$, A. Mampaso ${ }^{3}$, M. Perinotto ${ }^{1}$, \\ D. L. Pollacco ${ }^{4}$, J. R. Walsh ${ }^{5}$, N. A. Walton ${ }^{6}$, and A. A. Zijlstra ${ }^{7}$ \\ 1 Dipartimento di Astronomia e Scienza dello Spazio, Universitá di Firenze, L.go E. Fermi 2, 50125 Firenze, Italy \\ 2 Isaac Newton Group of Telescopes, Apartado de Correos 321, 38700 Santa Cruz de La Palma, Canarias, Spain \\ 3 Instituto de Astrofísica de Canarias, c. vía Láctea s/n, 38200, La Laguna, Tenerife, Canarias, Spain \\ ${ }^{4}$ School of Pure and Applied Physics, Queen's University Belfast, Belfast BT7 9NN, Northern Ireland, UK \\ 5 ST-ECF, ESO, Karl-Schwarzschild-Strasse 2, 85748 Garching bei München, Germany \\ ${ }^{6}$ Institute of Astronomy, University of Cambridge, Madingley Road, Cambridge CB3 OHA, UK \\ 7 Physics Department, UMIST, PO Box 88, Manchester M60 1QD, UK
}

Received 28 March 2003 / Accepted 6 May 2003

\begin{abstract}
In the framework of our narrow-band survey of the Local Group galaxies, we present the results of the search for planetary nebulae (PNe) in the dwarf irregular galaxies IC 10, Leo A and Sextans A. Using the standard on-band/off-band technique, sixteen new candidate PNe have been discovered in the closest starburst galaxy, IC 10. The optical size of this galaxy is estimated to be much larger than previously thought, considering the location of the new PNe in an area of $3.6 \mathrm{kpc} \times 2.7 \mathrm{kpc}$. We also confirm the results of previous studies for the other two dwarf irregular galaxies, with the detection of one candidate PN in Leo A and another one in Sextans A. We review the number of planetary nebulae discovered in the Local Group to date and their behaviour with metallicity. We suggest a possible fall in the observed number of PNe when $[\mathrm{Fe} / \mathrm{H}] \ll-1.0$, which might indicate that below this point the formation rate of PNe is much lower than for stellar populations of near Solar abundances. We also find non-negligible metallicity effects on the [O III] luminosity of the brightest PN of a galaxy.
\end{abstract}

Key words. planetary nebulae: general - galaxies: individual: Leo A, Sextans A, IC 10

\section{Introduction}

Most of the galaxies in the Local Group (LG) are dwarf irregulars and spheroidals. These morphological types also represent the most numerous objects in the nearby universe, but can be studied in great detail only at the close distance of the LG. We are performing a narrow- and broadband filter survey, the Local Group Census, which is mainly aimed at studying all classes of emission-line populations in the LG. The first results were presented by Magrini et al. (2002, hereafter M02), Magrini et al. (2003), Wright et al. (2003), while the status of the project is described at http://www.ing.iac.es/ rcorradi/LGC. M02 presented the detection of planetary nebulae $(\mathrm{PNe})$ in Sextans B. Our next targets were the dwarf irregular galaxies IC 10 (morphological type IrIV according to van den Bergh 2000, hereafter vdB00), Leo A (IrV) and Sextans A (IrV), that we discuss in the present work.

Send offprint requests to: $\mathrm{R}$. Corradi, e-mail: rcorradi@ing.iac. es

* Based on observations obtained at the $2.5 \mathrm{~m}$ INT telescope operated on the island of La Palma by the Isaac Newton Group in the Spanish Observatorio del Roque de Los Muchachos of the Instituto de Astrofisica de Canarias.
IC 10 is a highly obscured galaxy $(E(B-V) \simeq 0.85$, Sakai et al. 1999) located at a low Galactic latitude $b=-3$.3. Its distance (660 kpc, Sakai et al. 1999) and its position (only $\simeq 18^{\circ}$ apart from M 31 on the sky) suggest a possible membership to the M 31 subgroup (vdB00). It is the only starburst galaxy in the LG, and the presence of a large number of $\mathrm{H}$ II regions (Hodge \& Lee 1990) proves that it is undergoing massive star formation. IC 10 is a rather small galaxy with an effective radius $r_{\mathrm{e}}=0.5 \mathrm{kpc}$ (de Vaucouleurs \& Ables 1965), only one half the effective radius of the Small Magellanic Cloud (SMC), whereas their luminosities are comparable. Its oxygen abundance is higher than that of $\operatorname{SMC}(12+\log (\mathrm{O} / \mathrm{H})=8.20$ compared with 7.98 in SMC; Skillman et al. 1989), showing a higher past rate of star formation. This galaxy is clearly resolved in stars on ground-based images, and a large number of Wolf-Rayet stars is known (Massey et al. 1992). The presence of a large foreground extinction due to its location in a direction close to the Galactic plane has prevented so far deep studies of the stellar populations. A first search for PNe was undertaken by Jacoby \& Lesser (1981, hereafter JL81), and resulted in the discovery of one PN.

Leo $\mathrm{A}$ is a small irregular galaxy $\left(M<9 \times 10^{7} M_{\odot}\right.$, Young \& Lo 1996) at a distance of $800 \mathrm{kpc}$ (Dolphin et al. 2002), now firmly considered as a member of the LG (vdB00). 
It contains both old and young population components (Tolstoy et al. 1998), the older one amounting to approximately $10 \%$ of the stellar population located near to the centre of the galaxy. Strobel et al. (1991) have detected several H II regions excited by hot stars and an unresolved emission-line object, probably a planetary nebula. Heavy element abundance of this object shows a very low metallicity ( $\sim 2.4 \%$ solar; Skillman et al. 1989). In this galaxy, JL81 discovered two candidate PNe.

The membership of the LG of Sextans A, located at the distance of 1.45 Mpc (Sakai et al. 1996), is instead doubtful. It could form a possible group with NGC 3109, Antlia and Sextans B (vdB00). Sextans A seems to contain a conspicuous intermediate-age population, as suggested by its prominent red giant branch (Dohm-Palmer et al. 1997). Star formation, at present, is concentrated in a H II region complex observed by Hodge et al. (1994). Skillman et al. (1989) found an oxygen abundance $\sim 3 \%$ of Solar. A PN was identified by JL81.

In this paper we present [O III] and $\mathrm{H} \alpha+[\mathrm{N} \mathrm{II}]$ continuumsubtracted images of these three galaxies. These lead to the discovery of 16 new candidate PNe in IC 10. In Leo A, the two candidate PNe found by JL81 were shown instead to be normal stars, while the unresolved emission-line object seen by Strobel et al. (1991) is confirmed as a possible PN. We also detect a candidate PN in Sextans A, confirming the detection of JL81. Observations are described in Sect. 2. Data reduction and analysis are presented in Sect. 3. In Sect. 4, we discuss the results and in Sect. 5 we review the present knowledge about PNe in the LG, discussing their behaviour with metallicity. Summary and conclusions are given is Sect. 6.

\section{Observations}

Sextans A (DDO 75, 10h 11m00.5s -04d 41m 29s, J2000.0), Leo A (DDO 69, 09h 59m 26.4s +30d 44m 47s, J2000.0) and IC $10(00 \mathrm{~h} 20 \mathrm{~m} 23.2 \mathrm{~s}+59 \mathrm{~d} 17 \mathrm{~m} 30 \mathrm{~s}, \mathrm{~J} 2000.0)$ were observed using the prime focus wide field camera (WFC) of the $2.54 \mathrm{~m}$ Isaac Newton Telescope (La Palma, Spain), on February 2001, February 2002 and June 2002. The detector of the WFC is composed of four thinned EEV CCDs with $2048 \times 4096$ pixels each, with a pixel scale of $00^{\prime \prime} 33$. The large size of the field of view of the camera, 34' $\times 34^{\prime}$, allows to cover each galaxy in a single WFC pointing. The filters used are: [O III] (500.8/10.0 nm), H $\alpha+$ [N II] (656.8/9.5), Strömgren $y$ (550.5/24.0), $r^{\prime}$ (Sloan $\left.r, 624.0 / 134.7\right)$. The Strömgren $y$ and $r^{\prime}$ filters were used as off-band images for continuum subtraction of [O III $]$ and $\mathrm{H} \alpha+[\mathrm{N} \mathrm{II}]$ images, respectively.

Each exposure was split into three sub-exposures. The total exposure times were $3600 \mathrm{~s}$ for [O III] and $\mathrm{H} \alpha+[\mathrm{N} \mathrm{II}]$ for all galaxies. We also exposed for $3600 \mathrm{~s}$ in Strömgren $y$, except for Sextans A for which we exposed for $1800 \mathrm{~s}$. The exposure times in the $r^{\prime}$ filter were $1200 \mathrm{~s}$ for Leo A and Sextans A, and $1800 \mathrm{~s}$ for IC 10. The nights of February 2002 were not photometric, thus short exposures of IC 10 and of the Galactic PN IC 5117 (Wright et al., in preparation) were taken on June 2002 to allow absolute flux calibration. The nights of February 2001 were however photometric and several observations of the spectrophotometric standard stars: BD+33 2642 and G191-B2B (Oke 1990) were made each night.

\section{Data reduction and analysis}

Data reduction was done using IRAF ${ }^{1}$. The frames were debiased, flat-fielded, and linearity-corrected using the ING WFC data-reduction pipeline (Irwin \& Lewis 2001). Then we corrected for geometrical distortions and aligned all frames to the $[\mathrm{O}$ III] one. The sky background, measured in regions far from the galaxies, was subtracted from each frame. During the observations of Leo A the seeing was 1.' 3 through all filters. For Sextans A, it was 0.' 9 in the $\mathrm{H} \alpha+[\mathrm{N}$ II] and Strömgren $y$, and 1.' 1 in the [O III] and $r^{\prime}$ filters. For IC 10, it was 1.' 1 in the $\mathrm{H} \alpha+[\mathrm{N}$ II $]$ and Strömgren $y$ and 1.' 3 in the [O III] and $r^{\prime}$ filters.

We used the standard on-band/off-band technique (cf. Magrini et al. 2000) to identify emission-line objects. The $r^{\prime}$ and Strömgren $y$ frames, properly scaled, were subtracted from the $\mathrm{H} \alpha+[\mathrm{N}$ II] and [O III] frames, respectively. The flux of the emission-line objects were computed in the continuumsubtracted frames, using the IRAF task APPHOT. For the [O III] line, fluxes were transformed to equivalent $V$-mag magnitudes using $m_{[\mathrm{O} \text { III] }}=-2.5 \log F_{[\mathrm{O} \text { III] }}-13.74$ (Jacoby 1989).

The errors of the [O III $]$ and $\mathrm{H} \alpha+[\mathrm{N}$ II $]$ fluxes of the candidate PNe in Sextans A and Leo A, including photon statistics, background and flux calibration uncertainties, amount to a few percent. The fluxes of PNe in IC 10 have typical errors of $\sim 10 \%$ for PNe with $m_{[\mathrm{O} \text { III] }}<24.0,15-20 \%$ for $24.0<m_{[\mathrm{O} \text { III }]}<25.0$ and of $30 \%$ or more for the faintest PNe. These errors take into account photon statistics and the error on the zero point (2-3\%) for the flux calibration.

The instrumental magnitudes were calibrated by convolving the spectrum of the spectrophotometric standard star (Oke 1990) or the PN IC5117 (Wright et al., in preparation) with the response curve of each filter.

The astrometric solutions were computed using the IRAF tasks CCMAP and CCTRAN, using the USNO A2.0 catalogue (Monet et al. 1998) for IC 10 and the APM POSS1 for Sextans A and Leo A. The accuracy of all solutions is $\sim 0 ! 3 \mathrm{rms}$

\section{Candidate planetary nebulae}

Candidate PNe were identified using the same criterion as in Magrini et al. (2000), i.e. as spatially unresolved emissionline objects detected in the [O III] and $\mathrm{H} \alpha+[\mathrm{N}$ II] continuumsubtracted frames. The candidate $\mathrm{PNe}$ are listed in Table 1, with their positions, their [O III] and $\mathrm{H} \alpha+[\mathrm{N} \mathrm{II}]$ fluxes and the equivalent $V$-mag $m_{\text {[O III] }}$. Fluxes in the [O III] line at $\lambda=500.7 \mathrm{~nm}$ were corrected for the contribution of the companion oxygen line at $\lambda=495.9 \mathrm{~nm}$. This contribution varies depending on the different heliocentric radial velocities of the galaxies $\left(-348 \mathrm{~km} \mathrm{~s}^{-1}\right.$ for IC $10,20 \mathrm{~km} \mathrm{~s}^{-1}$ for Leo A, $324 \mathrm{~km} \mathrm{~s}^{-1}$ for Sextans A from the Nasa Extragalactic Database) and amounts to $6 \%, 11 \%$ and $14 \%$ respectively.

\footnotetext{
${ }^{1}$ IRAF is distributed by the National Optical Astronomy Observatories, which is operated by the Association of Universities for Research in Astronomy, Inc. (AURA) under cooperative agreement with the National Science Foundation.
} 
Table 1. PN candidates in IC 10, Leo A and Sextans A. [O III $] 500.7$ and $\mathrm{H} \alpha+[\mathrm{N} \mathrm{II}]$ observed fluxes are given in units of $10^{-16} \mathrm{erg} \mathrm{cm}^{-2} \mathrm{~s}^{-1}$. [O III] fluxes are corrected for the contribution of the companion oxygen line at $495.9 \mathrm{~nm}$ (see text). $R_{\text {corr }}=[\mathrm{O}$ III $] /(\mathrm{H} \alpha+[\mathrm{N} \mathrm{III}])$ is computed after correcting the fluxes for the reddening with $E(B-V)=0.85$ for IC 10, 0.015 for Sextans A, 0.02 for Leo A(vdB00), following Mathis' prescription (1990).

\begin{tabular}{lrrrrrr}
\hline \hline Identification & \multicolumn{2}{c}{ RA $(2000.0)$ Dec } & $F_{[\mathrm{OIII}]}$ & $F_{\mathrm{H} \alpha}$ & $m_{[\mathrm{O} \text { III] }}$ & $R_{\text {corr }}$ \\
\hline IC10 PN1 & $0: 18: 44.42$ & $59: 17: 47.7$ & 7.17 & 6.09 & 24.12 & 2.8 \\
IC10 PN2 & $0: 19: 04.32$ & $59: 17: 04.8$ & 7.95 & 3.68 & 24.01 & 5.2 \\
IC10 PN3 & $0: 19: 13.60$ & $59: 14: 43.9$ & 11.8 & 8.46 & 23.58 & 3.5 \\
IC10 PN4 & $0: 20: 06.73$ & $59: 15: 21.5$ & 3.95 & 3.69 & 24.76 & 2.1 \\
IC10 PN5 & $0: 20: 17.26$ & $59: 15: 52.5$ & 5.12 & 2.63 & 24.49 & 4.9 \\
IC10 PN6 & $0: 20: 21.14$ & $59: 21: 26.3$ & 1.15 & 0.85 & 26.10 & 3.2 \\
IC10 PN7 & $0: 20: 22.22$ & $59: 20: 01.6$ & 66.1 & 150. & 21.71 & 1.1 \\
IC10 PN8 & $0: 20: 28.82$ & $59: 07: 21.4$ & 13.2 & 8.68 & 23.46 & 3.7 \\
IC10 PN9 & $0: 20: 32.08$ & $59: 16: 01.6$ & 13.5 & 7.67 & 23.43 & 4.4 \\
IC10 PN10 & $0: 20: 40.98$ & $59: 18: 49.4$ & 14.8 & 7.01 & 23.33 & 5.2 \\
IC10 PN11 & $0: 20: 42.89$ & $59: 16: 30.6$ & 13.5 & 8.65 & 23.43 & 3.9 \\
IC10 PN12 & $0: 20: 46.28$ & $59: 21: 04.5$ & 3.45 & $<1$ & 24.66 & - \\
IC10 PN13 & $0: 20: 49.09$ & $59: 18: 32.9$ & 2.12 & 2.53 & 25.44 & 2.0 \\
IC10 PN14 & $0: 20: 50.96$ & $59: 20: 23.6$ & 6.07 & 2.17 & 24.30 & 7.0 \\
IC10 PN15 & $0: 21: 09.87$ & $59: 16: 58.9$ & 17.1 & 8.96 & 23.18 & 4.7 \\
IC10 PN16 & $0: 21: 11.23$ & $59: 16: 11.2$ & 4.96 & 11.9 & 24.52 & 1.1 \\
LEOA PN1 & $9: 59: 31.66$ & $30: 45: 28.0$ & 65.0 & 65.0 & 21.73 & 1.1 \\
SEXA PN1 & $10: 11: 01.58$ & $-4: 41: 22.9$ & 33.1 & 63.1 & 22.46 & 0.5 \\
\hline
\end{tabular}

\subsection{Planetary nebulae in IC 10}

Sixteen new candidate PNe were identified in the galaxy IC 10. This is the first identification of PNe in IC 10, except for one PN discovered by JL81, whose position was not reported. Our candidate $\mathrm{PNe}$ are detected both in the [O III] and $\mathrm{H} \alpha+[\mathrm{N} \mathrm{II}]$ continuum-subtracted images, so they cannot be confused with highly redshifted background galaxies. For each PN, we compute the $R=[\mathrm{O} \mathrm{III}] /(\mathrm{H} \alpha+[\mathrm{N}$ II] $)$ flux ratio after correcting for a mean foreground extinction $E(B-V)=0.85$ (vdB00) following Mathis (1990). All candidate PNe have values of the excitation index $R$ between 1.1 and 7.0, which is comparable with values for Galactic PNe (cf. Magrini et al. 2000, Fig. 3). The PNe distribution looks spatially uniform, and no bias against high/low $R$ or faint/bright PNe can be detected.

The PNe lie in a large area of $18.7 \times 14.1$ (see Fig. 1), that corresponds to a linear size of $3.6 \mathrm{kpc} \times 2.7 \mathrm{kpc}$ for a distance of $660 \mathrm{kpc}$ (Sakai et al. 1999). No PNe are found very close to the centre of this starburst galaxy, presumably because of the presence of numerous extended $\mathrm{H}$ II regions that cover a large fraction of that area. Eight PNe are situated around the starburst region, within $0.9 \mathrm{kpc}$ to the centre, the closest ones (PN4 and PN8) being at $\sim 0.35 \mathrm{kpc}$. The remaining PNe are located in the outskirts of IC 10 , outside the $25 \mathrm{mag} \operatorname{arcsec}^{-2}$ diameter $(5.5 \times 7 ! 0$, or $1.1 \times 1.3 \mathrm{kpc}$; Massey \& Armandroff 1995). The farthest of our PNe (PN1) is at the distance from the centre of $12.7(2.4 \mathrm{kpc})$. On the other hand, the large extent of the gaseous component of IC 10 is well known. From $21-\mathrm{cm}$ line observations, Huchtmeier (1979) found the presence of an enormous neutral hydrogen envelope $\left(62^{\prime} \times 80^{\prime}\right)$ surrounding the galaxy. PNe have proven to be excellent tracers of stellar populations in large volumes with a relatively low density of stars, whose integrated stellar light is low or even hardly detectable, like the intergalactic and intracluster space and in the haloes of elliptical galaxies (Arnaboldi et al. 2002). Thus, if spectroscopic studies confirm the nature as PNe of our candidates as well as their belonging to IC 10 (via their radial velocities), they would reveal the presence of a conspicuous stellar population at galactocentric distances much larger than considered so far. Moreover, Shostak \& Skillman (1989) found that both the outer hydrogen envelope and the core, which coincides with the small optical size of the galaxy, show a rotation along the same axis, but with opposite sign. It would be extremely interesting to verify whether this kinematical dichotomy also applies to PNe and to the stellar populations that they represent.

\subsubsection{Population size and completeness limit}

The number of candidate PNe detected in IC 10 is roughly consistent with the expected population size of this galaxy and its large foreground extinction $(E(B-V)=0.85$; vdB00). The population size can be estimated using a model describing a simple (i.e. coeval and chemically homogeneous) stellar population (Renzini \& Buzzoni 1986). As described by M02, the number of stars $n_{j}$ in any post-main-sequence phase $j$ is given by

$n_{j}=\dot{\xi} L_{\mathrm{T}} t_{j}$

where $\dot{\xi}$ is the specific evolutionary flux (number of stars per unit luminosity leaving the main sequence each year), $L_{\mathrm{T}}$ the total luminosity of the galaxy, and $t_{j}$ the duration of the evolutionary phase $j$ ( $\leq 20000$ yrs for the PN phase). Using the values of the parameters as in $\mathrm{M} 02$, the corresponding population size of IC 10 is between 50 and 200 objects considering the whole PN phase of $20000 \mathrm{yrs}$, and between 10 and 50 considering the phase during which the PN is bright enough to be observed at the detection limit of our survey, typically several thousand yrs. Taking into account that the central region $\sim 4^{\prime} \times 4^{\prime}$ is covered by a large complex of $\mathrm{H}$ II regions and 


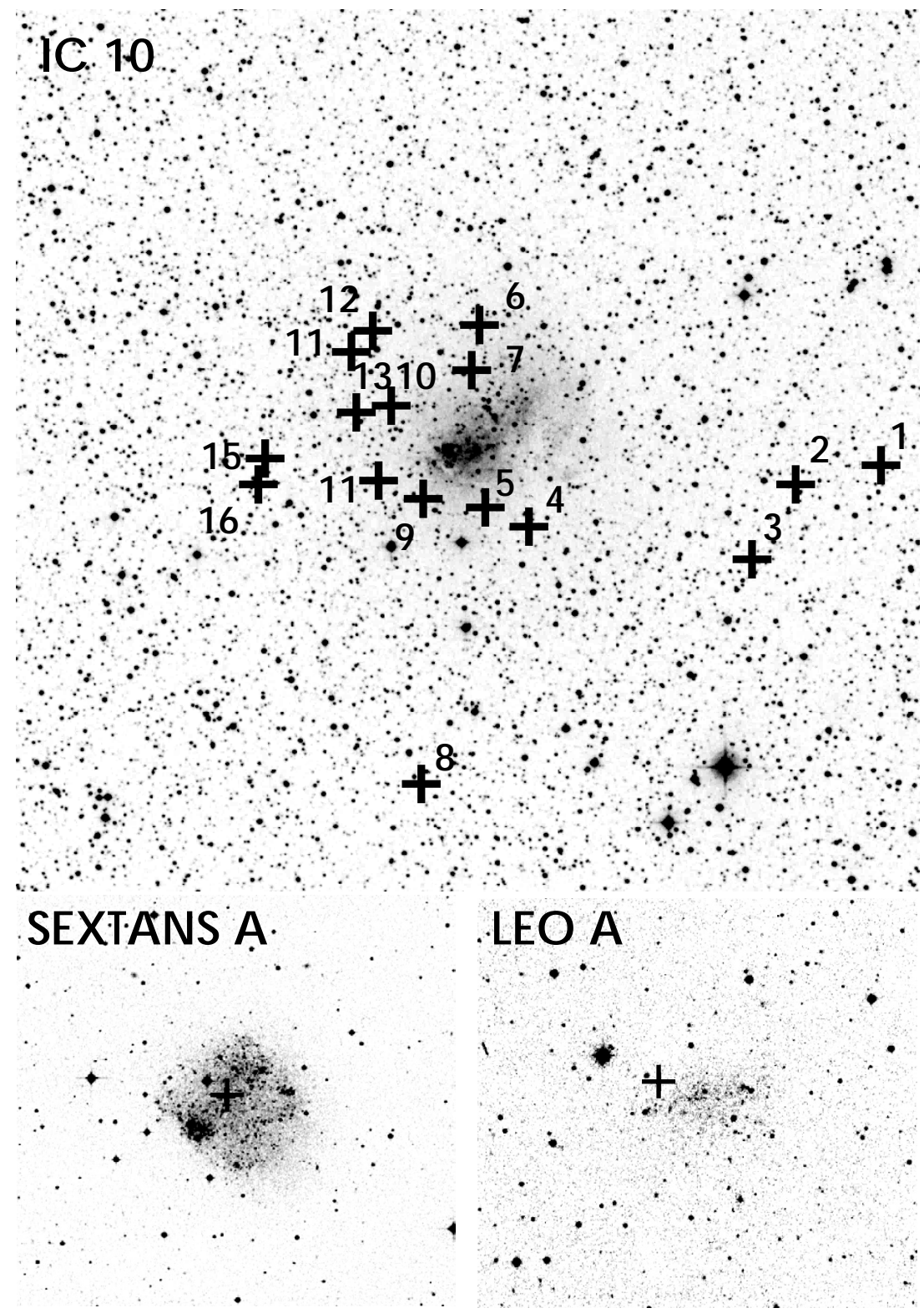

Fig. 1. Digitized Sky Survey images of the galaxies IC 10, Leo A and Sextans A. The size of the IC 10 frame is $30^{\prime} \times 30^{\prime}$, of Leo A and Sextans A $14^{\prime} \times 12^{\prime}$. North is at the top, East to the left. Candidate PNe are marked with a cross and their id. number as in Table 1.

the large foreground extinction in the direction of IC 10, the number of 16 candidate $\mathrm{PNe}$ is consistent with the expected population size.

As shown in Fig. 2, the number of our candidate PNe in IC 10 is also only slightly smaller than expected by scaling its luminosity with that of the LMC, and normalizing with the known number of LMC PNe where the survey for PNe is the most complete. The expected detectable PN population for a galaxy with the $V$-band luminosity of IC 10 would in fact be $\sim 30$ PNe. Our survey might therefore be slightly less complete owing to the greater distance of IC 10 compared to the LMC, the large reddening, and the presence of the large central complex of H II regions. Particularly, the star formation regions contribute considerably to the $V$-band luminosity, but they are too young to produce planetary nebulae. Our method for estimating the number of $\mathrm{PNe}$ in a galaxy makes use of the $V$-band luminosity, without considering that the galaxy luminosity must depend on the star formation history. The "effective" $V$-band luminosity is thus lower than the total $V$-band luminosity and consequently the dashed line in Fig. 2 slightly overestimates the expected number of PNe in IC 10.

We have also analyzed the "incompleteness" of our survey as a function of $m_{[\mathrm{O} I I]}$ and of the distance to the centre of the galaxy, as described in M02. We estimated the number of "missing" PNe by adding "artificial stars" with various $\left.m_{[\mathrm{O}} \mathrm{III}\right]$, as expected for the PN population of IC 10, in both [O III] and Strömgren $y$ frames. The incompleteness (i.e. a recovery rate of artificial stars less than 50\%, Minniti \& Zijlstra 1997) is due to the probability of missing an object in the $[\mathrm{O}$ III $]$ or $\mathrm{H} \alpha+[\mathrm{N} \mathrm{II}]$ images and/or to the probability of a wrong identification of a star in the continuum images. We found that our survey of IC 10 is incomplete for emission-line objects with $m_{[\mathrm{O} \text { III] }}>24.5$ and 


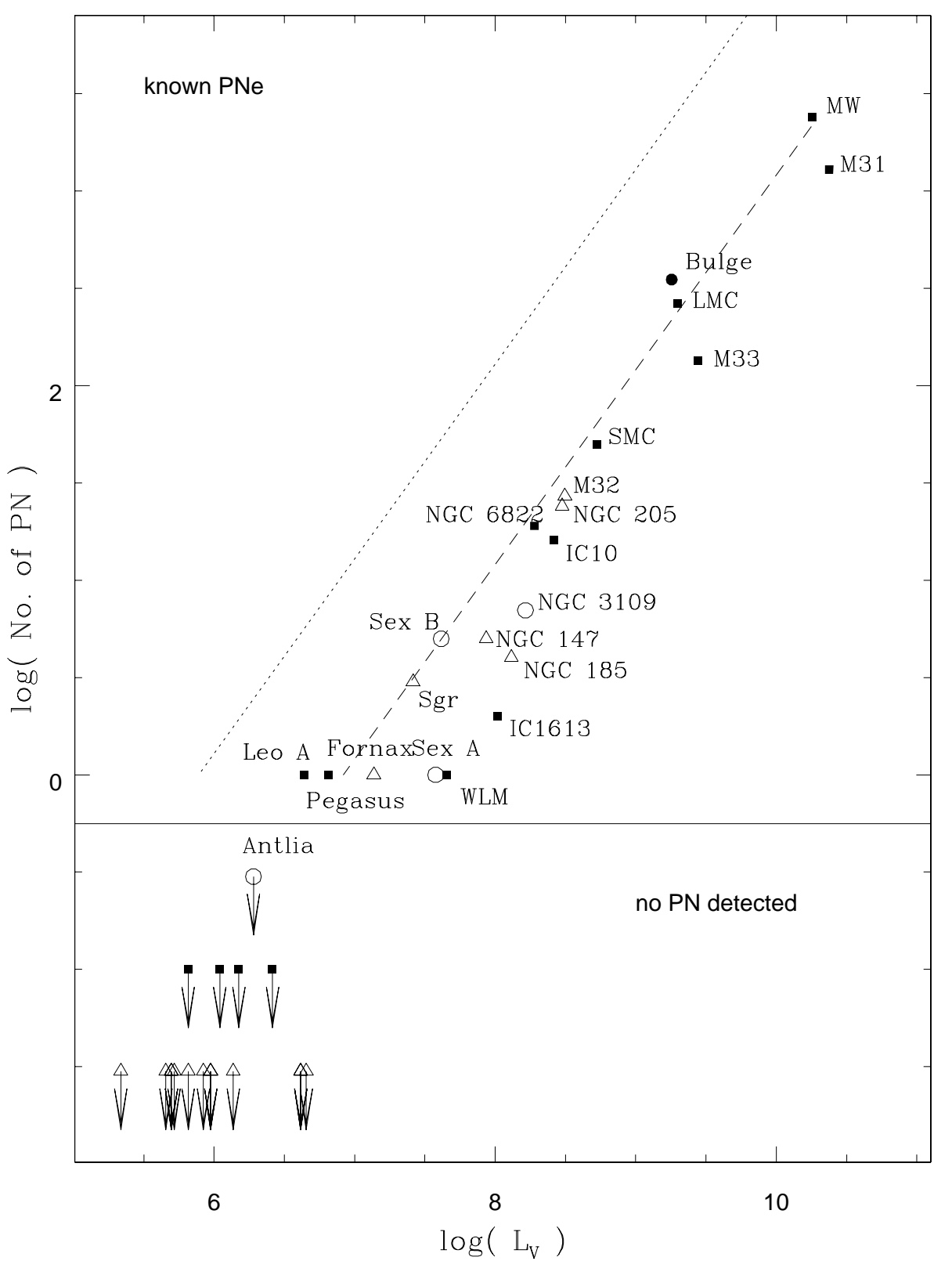

Fig. 2. The number of PNe in galaxies in or near the Local Group, versus the $V$-band luminosity in solar units. The dotted line shows the expected numbers based on a total number of PNe in the MW of 23000 . The dashed line is fitted to the known population in the LMC. Filled squares indicate LG gaseous galaxies, triangles indicate spheroidal galaxies and open circles show the NGC 3109 group.

there is a probability of $\sim 5 \%$ to find a star on the same line of sight and consequently to miss the emission-line object. The loss of faint emission-line objects is quite uniform across the whole field, whereas the probability of overlapping with a star or with a large $\mathrm{H}$ II region increases towards the centre, particularly in $\mathrm{H} \alpha+[\mathrm{N} \mathrm{II}]$ images where it reaches $40-50 \%$ within $4^{\prime}$ from the centre. Considering the fraction of the total mass inside the central $4^{\prime} \times 4^{\prime}$ region, we conclude that at least $5 \mathrm{PNe}$ brighter than the completeness limit may have been missed there.

Previous estimates of the distance to IC 10 locate it to a distance ranging from 0.5 to $3 \mathrm{Mpc}$ (Roberts 1962; de Vaucouleurs \& Ables 1965; Wilson et al. 1996; Tikhonov 1999; Sakai et al. 1999). The planetary nebulae luminosity function (PNLF) is widely used as an extragalactic distance indicator
(Jacoby 1989). When the PN population size is small, the absolute magnitude of the bright cut-off ( -4.53 for a large sample of $\mathrm{PNe}$, Ciardullo et al. 2002) of the luminosity function increases because the brightest PNe could not be observed (Méndez et al. 1993). In this case the PNLF cannot be used as a distance indicator. The $\left.m_{[\mathrm{O}} \mathrm{III}\right]$ of the brightest $\mathrm{PN}$ can give, however, an upper limit to the distance of IC 10, 1.8 Mpc. In Sect. 5 we will discuss how the metallicity correction applies to the $m_{\text {[O III] }}$ of the brightest PN.

\subsection{Planetary nebulae in Leo $A$}

Our observations identified one candidate PN in Leo A (see Table 1 and Fig. 1). The survey by JL81 allowed the discovery of two candidate PNe, which are shown in Fig. 2 of their paper. 


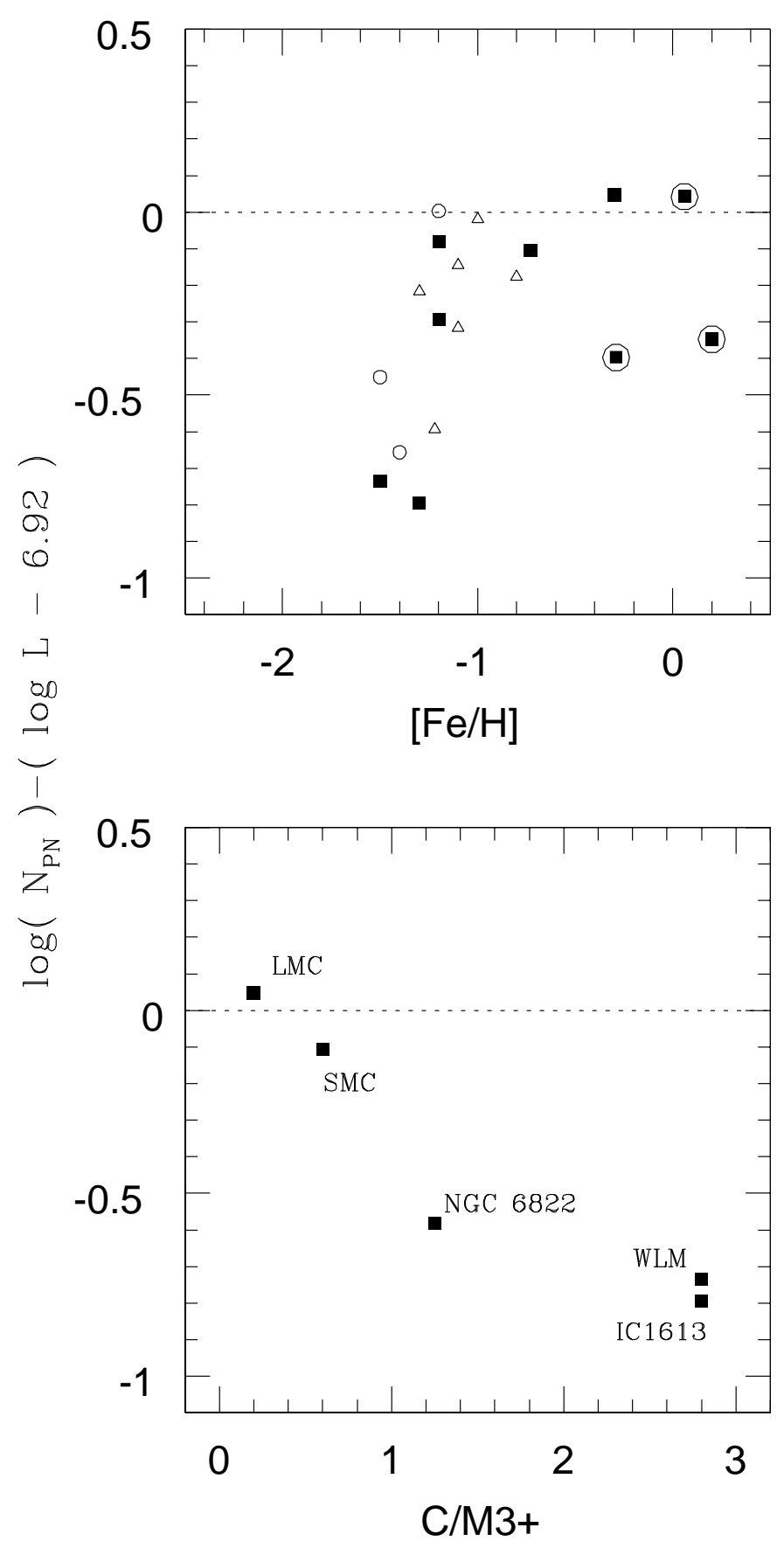

Fig. 3. The ratio between the observed and expected number of PNe $\left(\log N_{\mathrm{PN}}-\left(\log \left(L_{V}\right)-6.92\right)\right.$, see text) as a function of the metallicity of the galaxy (upper panel) or the ratio between carbon and $\mathrm{M}$ stars (lower panel). Squares are gas-rich local group galaxies, encircled squares are the three main LG spirals (M33, the Milky Way and M 31). Triangles are gas-poor spheroidal types and circles the galaxies probably not bound to the LG. In the lower panel only dwarf galaxies are shown.

These two candidate appear clearly both in our continuum and emission-line frames, therefore we conclude that they are normal stars. During an H $\alpha$ survey of Leo A, Strobel et al. (1991) have detected three H II regions and an unresolved emission region that they identified as a $\mathrm{PN}$. This object corresponds to our candidate PN. A spectrum of this object was obtained by Skillman et al. (1989), confirming its nature as a PN. From our [O III] and $\mathrm{H} \alpha+[\mathrm{N}$ II] fluxes, corrected for the foreground extinction $E(B-V)=0.02$ (Strobel et al. 1991), we computed an excitation index $R=1$, typical of a relatively low excitation PN, and/or of low oxygen abundance. This is lower than the value $R=1.4$ computed using the line fluxes in the spectrum by Skillman et al. (1989). Note however that their data would also measure an unrealistic $\mathrm{H} \alpha / \mathrm{H} \beta$ ratio of 2.3, suggesting that line fluxes in the blue region are overestimated. In fact, adjusting their line fluxes in the blue to achieve the theoretical value $\mathrm{H} \alpha / \mathrm{H} \beta=2.85$, [O III] would be scaled so as to provide $R=1.1 \pm 0.2$, in good agreement with our value.

Using Eq. (1), the expected PN population of Leo A is 1 to $3 \mathrm{PNe}$, thus consistent with the single PN observed. The upper limit to the distance modulus to Leo A given by the $m_{[\mathrm{O} \text { III] }}$ of the PN, without correcting for its low metallicity, is $\sim 26.3$ $(1.8 \mathrm{Mpc})$, very close to the distance obtained by Sandage (1986) using the three brightest stars in Leo A, but both methods are not reliable for galaxies with such a small population size. Our survey is fairly complete up to $m_{[\mathrm{O} \text { III] }}=24.5$ and no PN brighter than 24.5 mag remains to be discovered. Further deep spectroscopic study, as the one by Skillman et al. (1989) who measured the $\mathrm{O} / \mathrm{H}$ abundance, will give fundamental information about the metal content in one of the lower metal abundance galaxies of the Local Group.

\subsection{Planetary nebulae in Sextans A}

Several emission-line objects are shown in the [O III] and $\mathrm{H} \alpha+[\mathrm{N}$ II] continuum subtracted images of Sextans A. The extended emission-line sources are H II regions previously studied by Hodge et al. (1994), Dohm-Palmer et al. (1997) and supergiant ionized filaments studied by Hunter \& Gallagher (1997). We identified one unresolved emission-line source present both in the [O III] and $\mathrm{H} \alpha+[\mathrm{N}$ II] continuum-subtracted images (see Table 1 and Fig. 1). This candidate PN was previously discovered by JL81, and the [O III] flux presented in their paper is in agreement, within the errors, with our measure. The $R$ ratio for this candidate $\mathrm{PN}$, computed after correcting for reddening, $E(B-V)=0.015(\mathrm{vdB} 00)$, is $R=0.5$, indicating that either that it is a low-excitation PN, a compact $\mathrm{H}$ II region or that it has low oxygen abundance, as we can expect from the low metallicity of Sextans A $(12+\log (\mathrm{O} / \mathrm{H})=7.49$ from Skillman et al. 1989). It lies near to the centre of the galaxy and has $\left.m_{[\mathrm{O}} \mathrm{III}\right]=22.46$ which sets an upper limit to its distance modulus of $26.7(2.5 \mathrm{Mpc})$, without correction for low-metallicity. The completeness of our survey is up to $m_{[\mathrm{O} \text { III] }}=24.5$. No $\mathrm{PN}$ brighter than the $24.5 \mathrm{mag}$ remain to be discovered.

Sextans A and Sextans B have similar distances and are separated on the sky by only $10^{\circ} .4$. Considering also their small velocity difference $\left(23 \pm 6 \mathrm{~km} \mathrm{~s}^{-1}\right.$, vdB00), it is quite possible that they had formed together and then drifted apart over a Hubble time (vdB00). Their $V$-band luminosities and their mass are also similar, and thus their expected $\mathrm{PN}$ populations would be alike. Five PNe were discovered in Sextans B (M02), while only one candidate PN is detected in Sextans A. Statistically, this difference is only marginally 
significant, but may suggest some differences in their star formation history. In fact, M02 argued that Sextans B has a large number of intermediate-age stars formed in the last 5 Gyrs, while Sextans A has a stronger main sequence population (lowand intermediate-mass stars, Dohm-Palmer et al. 1997) likely due to a stronger recent star formation.

\section{Planetary nebulae in the Local Group}

A total of 2500 extragalactic PNe have been discovered over the last thirty years in almost all the LG galaxies whose total luminosity implies a population size large enough to allow the presence of PNe (cf. Ford et al. 2002; Magrini et al. 2002).

\subsection{The PN population size}

Figure 2 shows the number of PNe discovered to date in galaxies of the LG or in nearby groups, versus the $V$-band luminosity in solar units. This is an updated version of Fig. 2 in M02 and includes the PNe discovered in this paper (16 in IC 10 and 1 in Sextans A), as well as 19 PNe in NGC 6822 confirmed spectroscopically by P. Leisy (in preparation), 1284 candidate PNe in M 31 (D. Carter, private communication, see also Ford et al. 2002), one PN in WLM (Zijlstra, private communication), 2389 PNe in the Milky Way (Acker et al. 2002), 2 in IC 1613 (Magrini et al., in preparation). Compared to the previous version of this plot, the overall dispersion of data is reduced and a better relationship between the number of $\mathrm{PNe}$ and the $V$-luminosity of the galaxies can be seen. M 31 and M 33 are rather short of known objects, probably because of extinction effects and incompleteness in the existing surveys.

We have also investigated possible metallicity effects on the number of PNe in a galaxy. The upper panel of Fig. 3 shows the $[\mathrm{Fe} / \mathrm{H}]$ abundance of the galaxies vs. $\left(\log N_{\mathrm{PN}}-\left(\log \left(L_{V}\right)-\right.\right.$ $6.92)$ ), which is the logarithm of the ratio between the observed number of PNe $\left(N_{\mathrm{PN}}\right)$ and the expected population size $\left(\log \left(L_{V}\right)-6.92\right.$, scaled from the data on Sextans B where M02 found that one PN is expected per $L_{V}=10^{6.92}$ solar luminosities). The adopted number of PNe per unit luminosity is consistent with the statement by vdB00 that 6 carbon (C) stars are found at $M_{V}=-10$, making the expected ratio between $\mathrm{PNe}$ and $\mathrm{C}$ stars approximately 0.1 . Given that PNe are bright until they become optically thin at the radius approximately of $0.05 \mathrm{pc}$, which occurs after $2500 \mathrm{yr}$ for a nebular expansion velocity of $20 \mathrm{~km} \mathrm{~s}^{-1}$, Fig. 3 implies that the carbon star life time must be ten times longer or $\sim 25 \times 10^{3}$ yrs, which is qualitatively correct. Note that in this paper we use two different relations for the number of PNe: Eq. (1) and $\log \left(L_{V}\right)-6.92$. Equation (1) derives the total number of expected $\mathrm{PNe}$, while $\log \left(L_{V}\right)-6.92$ is based on the observations (so excluding faint objects, confusion, etc.) and thus infers the expected observable number of PNe. Note that adopting a value different from 6.92 as the normalization factor would not affect the discussion, as it would only shifts the relation $\log \left(L_{V}\right)-6.92$ up or down.

Figure 3 shows that there is a slight tendency for the number of detected PN to decrease with metallicity, if M 31 and $\mathrm{M} 33$, whose total observable population size in uncertain, are excluded (encircled squares at bottom). In this graph, we have also excluded galaxies where the expected number of PNe is <1 PN (Leo A and Pegasus) and IC 1613 which still lacks a proper survey for PNe. In particular, in Fig. 3 there are some hints of a shortfall in the observed number of $\mathrm{PNe}$ for $[\mathrm{Fe} / \mathrm{H}] \ll-1.0$. As $[\mathrm{Fe} / \mathrm{H}]=-1.0$ corresponds to the point where the AGB wind is expected to be driven no longer by dust, but only by pulsations (Zijlstra 1999), the lack of PNe might suggest that below this point the PN formation rate is largely reduced. Note that Mira variables are only seen in globular clusters when $[\mathrm{Fe} / \mathrm{H}]>-1$, suggesting there is significant change in AGB evolution at this point.

The bottom panel of Fig. 3 shows the PN deficit against the ratio between carbon and $\mathrm{M}$ stars $(C / M 3+)$ for the galaxies where this information is available, taken from Cook et al. (1986). The number of $M$ stars here is those with spectral type M 3 or later. This number increase rapidly with lower metallicity. The trend in the figure confirms the suggestion in the top panel.

As a matter of caution, it should however be noticed that part of the deficit of detected $\mathrm{PNe}$ in metal poor galaxies might also depend on the technique that is generally used to find $\mathrm{PNe}$, i.e. [O III] $500.7 \mathrm{~nm}$ imaging, as the [O III] emission decreases for low abundances of this element, as shown in the LMC and SMC by Leisy (2003, in preparation). On the other hand, our detection technique makes use in addition of $\mathrm{H} \alpha+[\mathrm{N}$ II] images. We have considered also low- $R$ candidate, as the one discovered in Sextans A, thus reducing the probability to loose $\mathrm{PNe}$ in low-metallicity galaxies.

\subsection{On the brightest PNe of the Local Group}

The first studies of the PNLF showed no strong evidence for metallicity dependence (Jacoby et al. 1988; Jacoby et al. 1990). Theoretical models by Jacoby (1989) suggested that the dependence of the bright cutoff of the PNLF with metallicity is modest, $\propto Z^{0.5}$. This effect is relatively unimportant when metallicity differs from galaxy to galaxy by $30 \%$ or less, but it could lead to significant differences when a wider range of metallicities is considered, as in the case of the LG. Dopita et al. (1992) examined the effects of metallicity on the luminosity of PNe, modeling the variation of the [O III] magnitude $M_{[\mathrm{O} \text { III] }}$ with the oxygen abundance as

$\Delta M_{[\mathrm{O} \text { III }]}=0.928[\mathrm{O} / \mathrm{H}]^{2}+0.225[\mathrm{O} / \mathrm{H}]+0.014$,

assuming the solar abundance of the oxygen to be $12+$ $\log (\mathrm{O} / \mathrm{H})=8.87$ (Grevesse et al. 1996). Equation (2) indicates that $\left.M_{[\mathrm{O}} \mathrm{III}\right]$ is brightest when the PN oxygen abundance is near to solar, while it decreases for both metal-poor and metal-rich galaxies. This has been observed in relatively metal-poor galaxies $(\mathrm{SMC}, 12+\log (\mathrm{O} / \mathrm{H})=8.03$; NGC $300,8.35)$ by Ciardullo et al. (2002), but it has never been observed in high metallicity galaxies because in those galaxies solar-metallicity stars are also available and they define the bright cutoff of the PNLF.

The correction given in Eq. (2) is significant when the metallicity is much lower than solar. The LG is a good environment in which investigate this issue, as oxygen abundance spans over a large range: from 7.30 of Leo A to 9.0 of M 31 (from vdB00). With the data presented in this paper and in the 


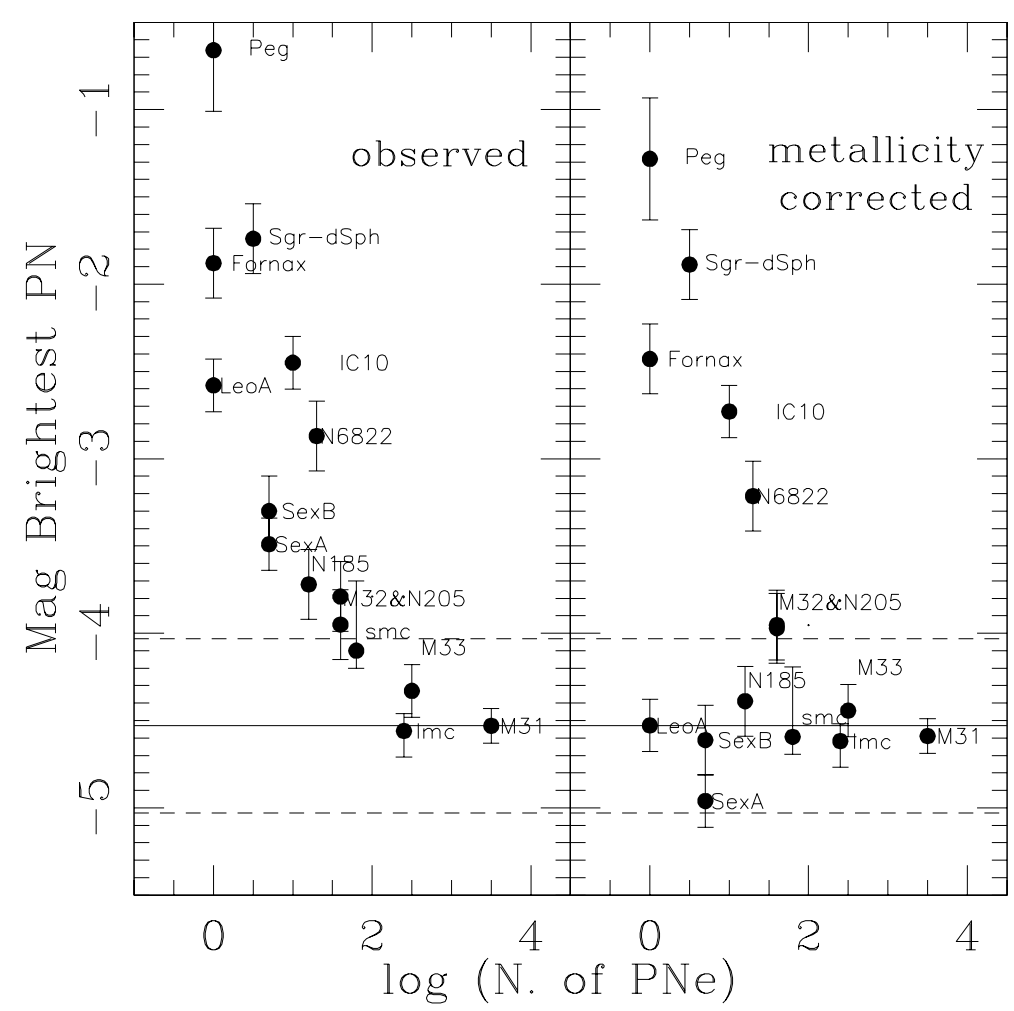

Fig. 4. The expected number of PNe vs. the absolute magnitude $\left(M_{*}\right)$ of the brightest PN, before (panel on the left) and after (panel on the right) metallicity correction following Dopita et al. (1992) prescription. For M 31, LMC and SMC we report the magnitude of the cutoff of their PNLFs with errors from Ciardullo et al. (2002). For M 33 the magnitude of the cutoff is from Durrell et al. (2003). The horizontal line show the magnitude of the PNLF cutoff $M_{*}=-4.53$ as expected for a galaxy with a large population size. Dashed lines mark 1-mag around $M_{*}=-4.53$.

previous surveys (Danziger et al. 1978; JL81; Killen \& Dufour 1982; Ciardullo et al. 1989; Morgan 1995; Zijlstra \& Walsh 1996; Leisy et al. 1997; M02; Durrell et al. 2003), an extensive sample of the brightest planetary nebulae of the LG is available. In Fig. 4, we present the PN population size versus $M_{*}$ or the cutoff of the PNLF (when available, i.e. M 31, M 33, SMC, LMC, cf. Ciardullo et al. 2002), Durrell et al. 2003 before and after correcting for metallicity using Eq. (2). Distances used to compute the absolute magnitudes are from vdB00, observed magnitudes are from the above cited papers. The solid horizontal line show the magnitude of the PNLF cutoff as expected for a galaxy with a large population size and Solar metallicity $\left(M_{*}=-4.53\right.$, Ciardullo 2002).

After correcting for metallicity using Eq. (2), a number of galaxies of the LG, especially those with a PNe population size larger than $10^{2}$, are tightly grouped around the "universal" value $M_{*}=-4.53$. This seems to confirm that the metallicity correction by Dopita et al. (1992) is at least qualitatively correct, and that the effects of metallicity are non-negligible for galaxies in the LG.

For galaxies with a population size of $10^{2}$ or less, $M_{*}$ is spread over a large range; it is likely that in these galaxies we start seeing the effect of having a small population size, as discussed in Sect. 4.1.2, together with some other effects, like for instance incompleteness of the existing surveys due to extinction or to the existence of areas of high stellar density or with large systems of H II regions (cf. e.g. with Sect. 4.1.1. for IC 10). In this respect, it is somewhat surprising to find small galaxies like Leo A, Sextans B, and Sextans A near $M_{*}=-4.53$, in spite of their very small PN population size.

The corrected magnitudes of the brightest PNe allow us to revise our upper limits to the distances of these galaxies presented previously in this paper and in M02. After correcting for metallicity, the upper limits to the distance moduli to Sextans B would be $25.5(1.3 \mathrm{Mpc}), 25.4(1.2 \mathrm{Mpc})$ for Sextans A, and $24.3(0.7 \mathrm{Mpc})$ for Leo A, in good agreement with other estimates (1.32 and 1.45 from vdB00, 0.8 from Dolphin et al. 2002), respectively, whereas for IC 10 the correction is not remarkable. We stress once more, however, that caution should be used when determining distance for such low-luminosity systems using PNe.

\section{Summary and conclusions}

In this paper, we have presented the discovery of sixteen new candidate $\mathrm{PNe}$ in IC 10, and confirmed two previously known candidate PNe, one in Leo A and the other one in Sextans A. These observations, together with other ones that will be presented in the future for other LG galaxies, provide a further improving in our understanding of the PN population of the Local Group. The behaviour of the numbers of PNe with galaxy metallicity has been discussed, finding a possible lack of PN when $[\mathrm{Fe} / \mathrm{H}] \ll-1.0$. The magnitudes of the brightest PNe of the LG have been reviewed and the possibility to measure 
distance using the magnitude of the brightest PN, after correcting for metallicity, has been discussed.

Future spectroscopic studies of individual objects will confirm their nature as PNe and allow detailed chemical abundance studies. In addition, they will provide unique information about the star formation history and chemical evolution of the parent galaxies, using tracers of stellar populations of intermediate to old age, and thus fully complementary to the information obtained from older (e.g. population II red giants) and younger (e.g. HII regions) classes of objects.

Acknowledgements. The data are made publically available through the Isaac Newton Groups' Wide Field Camera Survey Programme. This research has made use of the NASA/IPAC Extragalactic Database (NED), the APM and USNO-A2.0 Sky Catalogues, and the ESO Online Digitized Sky Survey. We would like to thank the anonymous referee for comments and suggestions that improved our paper.

\section{References}

Acker, A., Ochsenbein, F., Lundstroem, I., \& Parker, Q. 2003, Proc. of the Conf. Planetary Nebulae: Their Evolution and Role in the Universe, IAU Symp., 209, in press

Arnaboldi, M., Aguerri, J. A. L., Napolitano, N. R., et al. 2002, AJ, 123,760

Ciardullo, R., Jacoby, G. H., \& Ford, H. C. 1989, ApJ, 334, 715

Ciardullo, R., Feldmeier, J. J., Jacoby, G. H., et al. 2002, ApJ, 577, 31

Cook, K. H., Aaronson, M., \& Norris, J. 1986, ApJ, 305, 634

Danziger, I. J., Webster, B. L., Dopita, M. A., \& Hawarden, T. G. 1978, ApJ, 220, 458

de Vaucouleurs, G., \& Ables, H. 1965, PASP, 77, 272

Dohm-Palmer, R. C., Skillman, E. D., Saha, A., et al. 1997, AJ, 114, 2527

Dolphin, A. E., Saha, A., Claver, J., et al. 2002, AJ, 123, 3154

Dopita, M. A., Jacoby, G. H., \& Vassiliadis, E. 1992, AJ, 389, 27

Durrell, P. R., Ciardullo, R., Laychak, M. B., et al. 2003, AASM, 201, 13.11

Ford, H., Peng, E., \& Freeman, K. 2002, in The Dynamics Structures $\&$ History of Galaxies (San Francisco: ASP), ed. G. S. Da Costa, 41

Grevesse, N., Noels, A., \& Sauval, A. J. 1996, in Cosmic Abundances, ed. S. S. Holt, \& G. Sonneborn (San Francisco: ASP), ASP Conf. Ser., 99, 117

Hodge, P., \& Lee, M. G. 1990, PASP, 102, 26

Hodge, P., Kennicutt, R. C., \& Strobel, N. 1994, PASP, 106, 765

Huchtmeier, W. K. 1979, A\&A, 75, 170

Hunter, D. A., \& Gallagher, J. S. 1997, ApJ, 475, 65

Irwin, M., \& Lewis, J. 2001, New AR 45, Issue 1-2, 105

Jacoby, G. H. 1989, ApJ, 339, 39

Jacoby, G. H., \& Lesser, M. P. (JL81) 1981, AJ, 86, 185
Jacoby, G. H., Ciardullo, R., \& Ford, H. C. 1988, in The Astrophysical Distance Scale, ed. S. van den Bergh, \& C. J. Pritchet (Provo: Birgham Young Univ. Press), ASP Conf. Ser., 4, 42

Jacoby, G. H., Ciardullo, R., \& Walker, A. R. 1990, ApJ, 365, 471

Killen, R. M., \& Dufour, R. J. 1982, PASP, 94, 444

Leisy, P., Dennefeld, M., Alard, C., \& Guibert, J. 1997, A\&AS, 121, 407

Magrini, L., Corradi, R. L. M., Mampaso, A., \& Perinotto, M. 2000, A\&A, 355, 713

Magrini, L., Corradi, R. L. M., Walton, N. A., et al. (M02) 2002, A\&A, 386, 869

Magrini, L., Corradi, R. L. M., \& Munari, U. 2003 in the Proc. of the Conf. Symbiotic stars probing stellar evolution, ed. R. Corradi, J. Mikolajewska, \& T. J. Mahoney, ASP Conf. Ser., in press

Massey, P., \& Armandroff, T. E. 1995, AJ, 109, 2470

Massey, P., Armandroff, T. E., \& Conti, P. S. 1992, AJ, 103, 1159

Mathis, J. S. 1990, ARA\&A, 28, 37

Méndez, R. H., Kudritzki, R. P., Ciardullo, R., \& Jacoby, G. H. 1993 , A\&A, 275, 534

Minniti, D., \& Zijlstra, A. A. 1997, AJ, 114, 147

Monet, D., Bird, A., Canzian, B., et al. 1998, USNO-A2.0, (Washington DC: U.S. Naval Observatory)

Morgan, D. H. 1995, A\&AS, 112, 445

Oke, J. B. 1990, AJ, 99, 1621

Renzini, A., \& Buzzoni, A. 1986, in Spectral Evolution of Galaxies, ed. C. Chiosi, \& A. Renzini (Reidel), Ap. Space Sci. Lib., 122, 195

Roberts, M. S. 1962, AJ, 67, 431

Sakai, S., Madore, B. F., \& Freedman, W. L. 1996, ApJ, 461, 713

Sakai, S., Madore, B. F., \& Freedman, W. L. 1999, ApJ, 511, 671

Sandage, A. 1986, AJ, 91, 496

Shostak, G. S., \& Skillman, E. D. 1989, A\&A, 214, 33

Skillman, E. D., Kennicutt, R. C., \& Hodge, P. W. 1989, ApJ, 347, 875

Strobel, N. V., Hodge, P., \& Kennicutt, R. C. 1991, ApJ, 383, 148

Tikhonov, N. A. 1999, in The stellar content of Local Group galaxies, Proc. of the IAU Symp., 192 (San Francisco: ASP), ed. P. A. Whitelock, \& R. D. Cannon, 244

Tolstoy, E., Gallagher, J. S., Cole, A. A., et al. 1998, AJ, 116, 1244

van den Bergh, S. (vdB00) 2000, in The Galaxies of the Local Group (Cambridge: Cambridge University Press), 177

Wilson, C. D., Welch, D. L., Reid, I. N., Saha, A., \& Hoessel, J. 1996, AJ, 111, 1106

Wright, S., Greimel, R., Lennon, D. J., Corradi, R. L. M., \& Walton, N. 2003, in the Proc. of the Conf. A massive star odyssey, from main sequence to supernova, ed. K. A. van der Hucht, A. Herrero, \& C. Esteban, IAU Symp., 212, in press

Young, L. M., \& Lo, K. Y. 1996, ApJ, 462, 203

Zijlstra, A. A. 1999, in Asymptotic Giant Branch Stars, ed. T. Le Bertre, A. Lebre, \& C. Waelkens (San Francisco: ASP), IAU Symp., 191, 551

Zijlstra, A. A., \& Walsh, J. R. 1996, A\&A, 312, 21 